\title{
Molecular characterization of a novel polymycovirus from the phytopathogenic fungus Setosphaeria turcica
}

\section{Zhongnan Gao}

Yangtze University

Manyu Zhang

Yangtze University

Tingting Yu

Yangtze University

Xiaoyan Wang

Zunyi City Company, Guizhou Tobacco Company

\section{Xin Wang}

Fujian Agriculture and Forestry University

Hongliu An

Yangtze University

\section{Songbai Zhang ( $\nabla$ yangtze2008@126.com )}

College of Agriculture, Yangtze University https://orcid.org/0000-0002-8425-1434

Minghong Liu

Zunyi City Company, Guizhou Tobacco Company

\section{Shouguo Fang}

Yangtze University

\section{Annotated Sequence Record}

Keywords: Polymycovirus, Setosphaeria turcica, Setosphaeria turcica polymycovirus 1

Posted Date: February 16th, 2021

DOl: https://doi.org/10.21203/rs.3.rs-191401/v1

License: (1) (i) This work is licensed under a Creative Commons Attribution 4.0 International License.

Read Full License 


\section{Abstract}

A putative polymycovirus tentatively named Setosphaeria turcica polymycovirus 1 (StPmV1) was discovered from the phytopathogenic fungus Setosphaeria turcica. StPmV1 has a genome comprising 5 double stranded RNAs (dsRNAs). DsRNA1-3 each encodes a protein sharing significant but lower than $64 \%$ sequence identity with corresponding proteins from other polymycoviruses. DsRNA4-5 each encodes a protein with a sequence not conserved among polymycoviral proteins. However, the protein encoded by dsRNA4 is rich in proline $(P)$, alanine $(A)$, and serine $(S)$ residues, which is a feature shared by the socalled PAS-rich proteins encoded by all polymycoviruses. Phylogeny reconstruction using the RNAdependent RNA polymerases (RdRp) of accepted or putative polymycoviruses revealed that StPmV1 is most closely related to Plasmopara viticola lesion associated Polymycovirusmyco 1 (PvaPolymyco1), a putative polymycovirus recovered from the phytopathogenic oomycetes Plasmopara viticola. These data suggest that StPmV1 may represent a novel species of the genus Polymycovirus under the family Polymycoviridae. To our knowledge, this is the first polymycovirus reported from S. turcica.

\section{Introduction}

Polymycoviriruses constitute a family of double-stranded RNA (dsRNA) viruses that infect a wide range of fungi (https://talk.ictvonline.org/taxonomy). Unlike most other dsRNA viruses, polymycoviriruses do not form isometric virions [1-9]. Instead, they encode a protein overrepresented by proline (P), alanine (A), and serine (S) residues. In the case of Colletotrichum camelliae filamentous virus 1 (CcFV1), this PAS-rich protein (PASrp) encapsulates polymycoviriral dsRNAs into filamentous particles [3]. In most other cases, PASrp coats but not encapsulates viral dsRNAs [1-2,4-9]. Moreover, polymycoviriruses have a RNAdependent RNA polymerase (RdRp) more closely related to the RdRp of positive-sense single stranded RNA (+ ssRNA) viruses than to those encoded by other dsRNA viruses. At least some polymycoviriruses are infectious as naked genomic dsRNAs $[1,3]$. These features have led to the idea that polymycoviriruses may represent an evolutionary intermediate between dsRNA and + ssRNA viruses and between capsidated and capsid-less viruses $[1,3,8]$. Polymycoviruses show a great flexibility in genome arrangement. For example, Aspergillus fumigatus tetramycovirus-1 (AfuTmV-1), the type species of the family Polymycoviridae, has a genome consisting of 4 dsRNAs [1]. The genome of many other polymycoviriruses, however, have 1-3 additional dsRNAs, which encode proteins not conserved among polymycoviriruses [2-9]. Although the effects of most polymycoviriruses on the growth and pathogenicity of their host fungi remain poorly characterized, some polymycoviriruses have been found to confer hypo- or hypervirulence to their hosts [1-4].

Setosphaeria turcica is a fungal pathogen of corn as well as many other important crop plants. Four mycoviruses have been discovered from $S$. turcica, an ambiguivirus, a hypovirus, and a mitovirus, which were identified by searching the transcriptome data of $S$. turcica, and a fusarivirus, which was discovered by conventional cloning experiments [11]. In this sequence note, we report the 5 th mycovirus of $S$. turcica, a polymycovirirus tentatively named Setosphaeria turcica polymycovirus 1(StPmV1). 


\section{Provenance Of The Virus Material}

S. turcica strain ZY17 was isolated from a corn leaf showing northern leaf blight symptoms from Zaoyang, Hubei province of China, in 2019. After a growth of 6 days on a potato dextrose agar (PDA) plate at $28^{\circ} \mathrm{C}$, mycelia $(\sim 0.2 \mathrm{~g})$ of ZY17 were harvested and subjected to dsRNA extraction with a procedure as described previously [12]. The extract was electrophoresed in a $1 \%$ agarose gel. Four discrete bands were observed from the gel after Ethidium bromide (EB) staining (Fig. 1A). Assuming that dsRNAs have a migration speed comparable to that of dsDNAs, this banding pattern suggests the presence of multiple dsRNA elements sizing between 1 and $2.5 \mathrm{~Kb}$. To sequence these dsRNAs, each band was excised from the gel. DsRNA within each gel slice was recovered with the TIANgel Midi Purification Kit (Tiangen, China) and reverse transcribed with the tagged random primer 5 'CGATCGATCATGATGCAATGCNNNNNN-3'. The complementary DNA (cDNA) was amplified with the primer 5'-CGATCGATCATGATGCAATGC-3'. PCR amplicons longer than 500 bp were each ligated to the vector pMD-20T (TAKARA) and the recombinant plasmid was transformed to Escherichia coli DH5a. For each transformation, 8-14 randomly selected colonies were sent to Beijing Genomics Institute (BGI, China) for Sanger sequencing. The sequences were analyzed and assembled using DNASTAR. The 5 ' and 3 ' terminus of each assembled dsRNA were obtained by RNA ligase mediated rapid amplification of CDNA ends (RLM-RACE) [13]. The results showed that the smallest band was formed by two distinct dsRNAs, one has a size of $1179 \mathrm{bp}$, the other is 1115-bp in length. The 3 larger bands each represents a single dsRNA with a size of 2427, 2259 and $1994 \mathrm{bp}$, respectively. The 5 dsRNAs are named dsRNA1-5 according to the decreasing order of their size and their sequences were deposited in the GenBank under the accession numbers MW429374.1-MW429378.1. Overall, each nucleotide position on each dsRNA was sequenced at least 4 times.

\section{Sequence Properties}

In accordance with the observation that polymycoviruses have a genome rich in GC pairs, the GC\% content of StPmV1 dsRNA 1-5 are 60.90\%, 61.22\%, 61.08\%, 62.34\% and 62.06\%, respectively [1-9]. Using the ORF finder program available at National Coalition Building Institute (NCBI), a single open reading frame (ORF) was detected on each dsRNA. For simplicity, these ORFs are called ORF1-5 and the proteins predicted from them are named P1-P5, respectively. ORF1-5 are preceded by a 5'- untranslated region (UTR) of 23, 71, 52, 98, and $23 \mathrm{nt}$ and followed by a 3'-UTR of 100, 94, 97, 118 and $159 \mathrm{nt}$, respectively (Fig. 1B). Supporting the idea that dsRNA1-5 are genomic components of the same virus, their terminal sequences are very similar: the coding strand of all the 5 dsRNAs has an AU-rich $5^{\prime}$-terminus preceded by the dinucleotide GC and a GC rich 3'-terminus terminated with $4 \mathrm{U}$ nucleotides (Fig. 1C).

$\mathrm{P} 1$ has a molecular mass of $84.28 \mathrm{kDa}$. A search of the NCBI conserved domain database revealed a RdRp domain (cd01699, RNA_dep_RNAP), spanning amino acid positions 466-603 [14-16]. Like the situations observed from other polymycoviruses, this domain contains a GDNQ motif that is common to the RdRp of -ssRNA viruses of the order Mononegavirales (Fig. 2A) [1-9, 17]. These data suggest that P1 is the RdRp of StPmV1. Indeed, a BLASTp search of the NCBI protein database found that P1 shares an 
identity of $63.29 \%, 57.63 \%$ and $55.96 \%$ with the RdRp of Plasmopara viticola lesion associated Polymycovirusmyco 1 (PvaPolymyco1), PvaPolymyco2, Magnaporthe oryzae polymycovirus 1 (MoPmV1), and an identity of $25.53 \%-53.70 \%$ with the RdRp of other polymycoviruses $[9,18]$.

P2 has a molecular mass of $74.96 \mathrm{kDa}$. No conserved domain was found on P2. However, P2 shows $29.48 \%-47.49 \%$ sequence identity to dsRNA2-encoded proteins from many, although not all, polymycoviruses. The $\mathrm{N}$-terminus of $\mathrm{P} 2$ has a sequence reading MADLTRL. This sequence, as part of a signal peptide, is found at the N-termini of dsRNA2-encoded proteins for all polymycoviruses, as noted by Kotta-Loizou1and Coutts [4]. According to the proposition of Kotta-Loizou1and Coutts, polymycoviruses may use the protein encoded by dsRNA2 to anchor their replication machinery to a membranous compartment within the host cell [4]. Supporting this, a strong transmembrane helix, from amino acid residue 279 to residue 298, was identified from P2 using the TMpred server (data not shown) [19]. Notably, a hypothetical protein encoded by an unnamed dsRNA (GenBank accession ACL80752) from a fungus of the genus Alternaria showed $60.34 \%$ amino acid sequence identity with P2.

P3 has a molecular mass of $65.68 \mathrm{kDa}$. A Methyltransferase domain (pfam13649) was found on P3, spanning amino acid residue position 131 to position 236. This Methyltransferase domain has been detected from dsRNA3-encoded proteins of all polymycoviruses [1-9]. Although experimental data are lacking at present, the presence of this domain suggests that polymycoviruses may use dsRNA3-encoded proteins to cap their genomic or messenger RNAs [1]. Like P2, P3 shows significant similarities to cognate proteins from a subset of polymycoviruses. The most similar protein, encoded by dsRNA3 of PvaPolymyco1, shares an amino acid sequence identity of $59.54 \%$ with P3 [9].

P4 has a molecular mass of $33.57 \mathrm{kDa}$. This 320 -amino acid long protein has 31,38 and $43 \mathrm{P}, \mathrm{A}$ and $\mathrm{S}$ residues, respectively, suggesting that it is the PASrp of StPmV1. Although common to all polymycoviruses, PASrp does not have a conserved sequence $[1-9,20]$. Consistent with this, the BLASTp search using P4 as a query returned no significant hits. Interestingly, however, a provisional domain described as "large tegument protein UL36" (cl33720) was found on P4, spanning amino acid positions 6-229.

P5 has a molecular mass of $33.81 \mathrm{kDa}$. This protein does not show similarity to any known proteins, nor does it contain any conserved domains. Thus, the function of P5 cannot be predicted at present.

To investigate the evolution relationships between StPmV1 and other polymycoviruses, the RdRp sequences of accepted or putative polymycoviruses were submitted to Phylogeny.fr, an online web service that allows phylogeny reconstruction with a "One Click" mode [21-22]. The RdRp of Hadaka virus 1 (HadV1), a polymycovirus-related virus, was used as an outgroup. The maximum likelihood tree returned from Phylogeny.fr divided polymycoviruses into 4 groups (Fig. 2B). StPmV1 was placed in Group 2. As a comparison, AfuTmV-1, the type species of the family Polymycoviridae, was placed in Group 3 [1]. Beauveria bassiana polymycovirus 1 (BbPmV1), a polymycovirus infecting the entomopathogenic fungus Beauveria bassiana, was placed in Group 1. CcFV1, the only known polymycovirus forming filamentous virions, was placed in Group 4 [3]. Within Group 2, StPmV1 formed a monophyletic clade with 
PvaPolymyco1. Interestingly, PvaPolymyco3 and PvaPolymyco5, which were recovered from the same host as PvaPolymyco1, were not included in this clade [9].

Overall, StPmV1 has a genome with features characteristic to polymycoviruses. However, StPmV1 encodes a RdRp sharing $<64 \%$ sequence identity with the RdRp of other polymycoviruses. StPmV1 may infect $S$. turcica, a fungus from which no polymycovirus has been reported. These data suggest that StPmV1 represents a novel species of the genus Polymycovirus under the family Polymycoviridae. Because some polymycoviruses have been shown to affect the pathogenicity of their hosts, further studies on the effects of StPmV1 on S. turcica are interesting.

\section{Declarations}

\section{Acknowledgments}

This work was supported by the Science and Technology Project of Guizhou tobacco company (No. 201921), the National Natural Science Foundation of China (No. 31972243) and the Opening fund of Key Lab of Biopesticide and Chemical Biology, Ministry of Education, Fujian Agriculture and Forestry University. We are grateful to Dr. Zhenguo Du at Institute of Plant Virology, Fujian Agriculture and Forestry University for his help in preparing the manuscript.

\section{Compliance with Ethical Standards:}

\section{Conflict of Interest:}

All authors declare that they have no conflict of interest.

\section{Ethical approval:}

This article does not contain any studies with human participants or animals performed by any of the authors.

\section{References}

1. Kanhayuwa L, Kotta-Loizou I, Ozkan S, Gunning AP, Coutts RHA (2015) A novel mycovirus from Aspergillus fumigatus contains four unique dsRNAs as its genome and is infectious as dsRNA. Proc Natl Acad Sci USA 112:9100-9105

2. Zhai LF, Xiang J, Zhang MX, Fu M, Yang ZK, Hong N, Wang GP (2016) Characterization of a novel double-stranded RNA mycovirus conferring hypovirulence from the phytopathogenic fungus Botryosphaeria dothidea. Virology 493:75-85

3. Jia H, Dong KL, Zhou LL, Wang GP, Hong N, Jiang DH, Xu WX (2017) A dsRNA virus with filamentous viral particles. Nat Commun 8:168 
4. Kotta-Loizou I, Coutts RHA (2017) Studies on the virome of the entomopathogenic fungus Beauveria bassiana reveal novel dsRNA elements and mild hypervirulence. PLoS Pathog 13:e1006183

5. Niu YH, Yuan YZ, Mao JL, Yang Z, Cao QW, Zhang TF, Wang SQ, Liu DL (2018) Characterization of two novel mycoviruses from Penicillium digitatum and the related fungicide resistance analysis. Sci Rep 8:5513

6. Nerva L, Forgia M, Ciuffo M, Chitarra W, Chiapello M, Vallino M, Varese GC, Turina M (2019) The mycovirome of a fungal collection from the sea cucumber Holothuria polii. Virus Res 273:197737

7. Mahillon M, Decroës A, Liénard C, Bragard C, Legrève A (2019) Full genome sequence of a new polymycovirus infecting Fusarium redolens. Arch Virol 164:2215-2219

8. Sato Y, Jamal A, Kondo H, Suzuki N (2020) Molecular characterization of a novel polymycovirus from Penicillium janthinellum with a focus on its genome-associated PASrp. Front Microbiol 11:592789

9. Chiapello M, Rodríguez-Romero J, Ayllón MA, Turina M (2020) Analysis of the virome associated to grapevine downy mildew lesions reveals new mycovirus lineages. Virus Evol 6:veaa058

10. Sato Y, Caston JR, Suzuki N (2018) The biological attributes, genome architecture and packaging of diverse multi-component fungal viruses. Curr Opin Virol 33:55-65

11. Gao ZN, Cai LN, Liu MH, Wang XY, Yang JG, An HL, Deng QC, Zhang SB, Fang SG (2021) A novel previously undescribed fusarivirus from the phytopathogenic fungus Setosphaeria turcica. Arch Virol. DOI:10.1007/s00705-021-04954-x

12. Deng QC, Wang HR, Li C, Li P, Fang SG, Yang S, Yan F, Zhang SB, Chen Z (2017) The complete genomic sequence of a novel alphapartitivirus from Bipolaris maydis, the causal agent of corn southern leaf blight. Arch Virol 162:2433-2436

13. Liu X, Gorovsky MA (1993) Mapping the $5^{\prime}$ and $3^{\prime}$ ends of Tetrahymena thermophila mRNAs using RNA ligase mediated amplification of cDNA ends (RLM-RACE). Nucleic Acids Res 21:4954-4960

14. Lu SN, Wang JY, Chitsaz F, Derbyshire MK, Geer RC, Gonzales NR, Gwadz M, Hurwitz DI, Marchler GH, Song JS, Thanki N, Yamashita RA, Yang MZ, Zhang DC, Zheng CJ, Lanczycki CJ, Marchler-Bauer A (2020) CDD/SPARCLE: the conserved domain database in 2020. Nucleic Acids Res 48:D265-D268

15. Bruenn JA (1993) A closely related group of RNA-dependent RNA polymerases from double-stranded RNA viruses. Nucleic Acids Res 21:5667-5669

16. Koonin EV, Dolja VV (1993) Evolution and taxonomy of positive-strand RNA viruses: Implications of comparative analysis of amino acid sequences. Crit Rev Biochem Mol Biol 28:375-430

17. Poch O, Blumberg BM, Bougueleret L, Tordo N (1990) Sequence comparison of five polymerases (L proteins) of unsegmented negative-strand RNA viruses: Theoretical assignment of functional domains. J Gen Virol 71:1153-1162

18. Hu G, Kurgan L (2019) Sequence similarity searching. Curr Protoc Protein Sci 95:e71

19. Hofmann K, Stoffel W (1993) TMbase-A database of membrane spanning proteins segments. Biol Chem Hoppe-Seyler 374:166 
20. Sato Y, Shamsi W, Jamal A, Bhatti MF, Kondo H, Suzuki N (2020) Hadaka virus 1: a capsidless eleven-segmented positive-sense single-stranded RNA virus from a phytopathogenic fungus. Fusarium oxysporum mBio 11:e00450-e00420

21. Dereeper A, Guignon V, Blanc G, Audic S, Buffet S, Chevenet F, Dufayard JF, Guindon S, Lefort V, Lescot M, Claverie JM, Gascuel O (2008) Phylogeny.fr: robust phylogenetic analysis for the nonspecialist. Nucleic Acids Res 36:W465-W469

22. Dereeper A, Audic S, Claverie JM, Blanc G (2010) BLAST-EXPLORER helps you building datasets for phylogenetic analysis. BMC Evol Biol 10:8

\section{Figures}

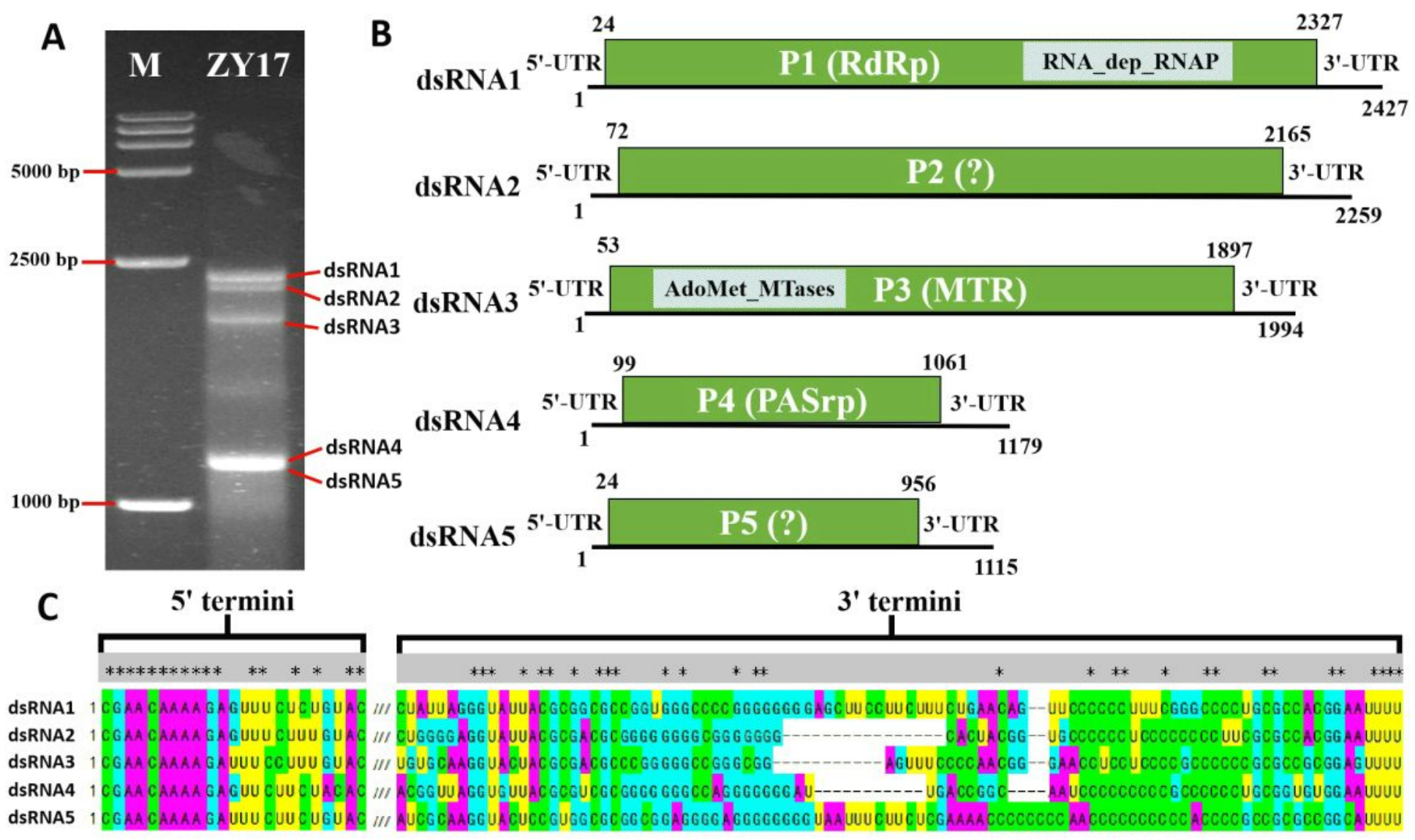

\section{Figure 1}

Identification and molecular characterization of Setosphaeria turcica polymycovirus 1(StPmV1). (A) Agarose gel electrophoresis of the dsRNAs extracted from Setosphaeria turcica strain ZY17. The positions of StPmV1 dsRNA 1-5 are indicated. M indicates a DNA marker (DL15000, TAKARA). (B) The genome organization of StPmV1. Each dsRNA is represented by a horizontal line. The open reading 
frame (ORF) detected on each dsRNA is shown by a green box and the nucleotide positions of the initiation and termination codon of each ORF are shown above the box. The relative positions of the conserved domains detected on each protein of StPmV1 are shown inside the boxes. (C) The 5'- and 3'temini of the coding strand of StPmV1 dsRNA 1-5.

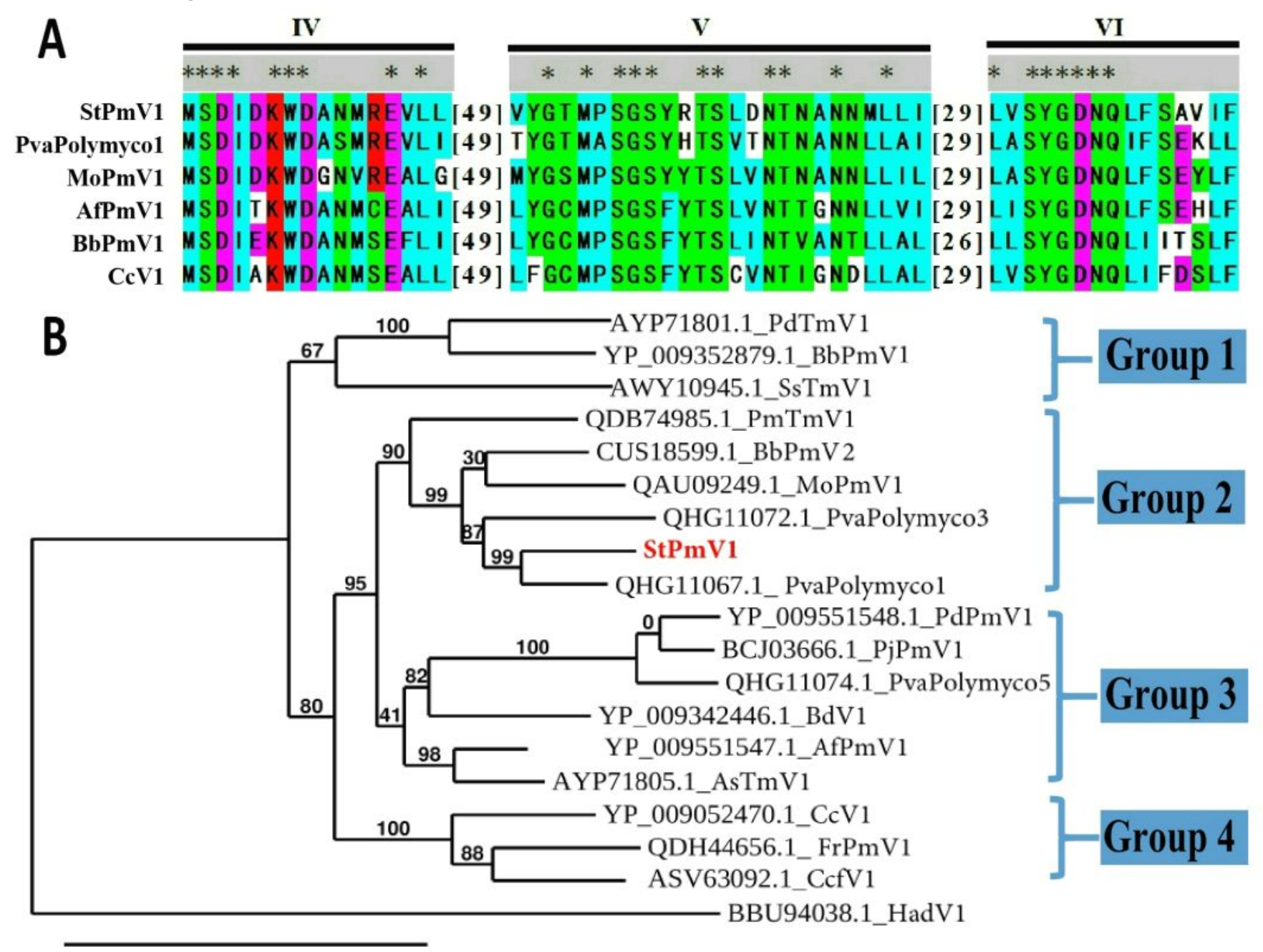

0.8

\section{Figure 2}

The evolution relationships between Setosphaeria turcica polymycovirus 1(StPmV1) and other polymycoviruses. (A) Conserved RdRp motifs detected on the protein encoded by dsRNA1 of StPmV1.

The alignment was performed with CLUSTAL_X. Three conserved RdRp motifs corresponding to motifs IV, $\mathrm{V}$, and VI were shown. The asterisks indicate identical amino acid residues. Colors indicate highly conserved or related residues. Numbers within the brackets indicated the number of amino acid residues not shown. (B) A maximum likelihood tree constructed using Phylogeny.fr, with the "One Click" mode. The RdRp sequences of polymycoviruses or putative polymycoviruses are used for phylogeny inference. Bootstrap values (\%) of each node are shown above the branch. The name of StPmV1 is colored in red. The names of other polymycoviruses and Hadaka virus 1 (HadV1), which was used as an outgroup, are black colored and preceded by the GenBank accessions of the RdRp of these viruses. The abbreviations used for viral names are as follows: Penicillium brevicompactum tetramycovirus 1 (PbTmV1), Beauveria 
bassiana polymycovirus 1 (BbPmV1), Sclerotinia sclerotiorum tetramycovirus 1 (SsTmV1), Phaeoacremonium minimum tetramycovirus 1 (PmTmV1), Beauveria bassiana polymycovirus 2 (BbPmV2), Magnaporthe oryzae polymycovirus 1 (MoPmV1), Plasmopara viticola lesion associated Polymycovirusmyco 3 (PvaPolymyco3), Plasmopara viticola lesion associated Polymycovirusmyco 5 (PvaPolymyco5), Plasmopara viticola lesion associated Polymycovirusmyco 1 (PvaPolymyco1), Penicillium digitatum polymycoviruses 1 (PdPmV1), Penicillium janthinellum polymycovirus 1 (PjPmV1), Botryosphaeria dothidea virus 1 (BdV1), Aspergillus fumigatus polymycovirus 1 (AfPmV1), Aspergillus spelaeus tetramycovirus 1 (AsPmV1), Cladosporium cladosporioides virus 1 (CcV1), Fusarium redolens polymycovirus 1 (FrPmV1), Colletotrichum camelliae filamentous virus 1 (CcfV1).

\section{Supplementary Files}

This is a list of supplementary files associated with this preprint. Click to download.

- StPmV1dsRNA1Submission2415166MW429374.1.txt

- StPmV1dsRNA2Submission2415172MW429375.1.txt

- StPmV1dsRNA3Submission2415174MW429376.1.txt

- StPmV1dsRNA4Submission2415177MW429377.1.txt

- StPmV1dsRNA5Submission2415180MW429378.1.txt 